\title{
Algorithm for Route Planning via Facilities with Time Dependent
}

\author{
Pramod Singh Rathore \\ M.Tech, Department of C.S \\ Govt. Engg. College, Ajmer \\ (Rajasthan), India
}

\author{
Atul Chaudhary \\ M.Tech, Department of C.S \\ Govt. Engg. College, Ajmer \\ (Rajasthan), India
}

\begin{abstract}
In today's century, people are looking for faster and efficient communication for travelling from source to destination via facilities. As the human tendency always try to go from source to cover or attend some facilities and then reach to destination within a required time. This paper focus on algorithm for optimal route planning via facility ( i.e petrol, grocery etc) with time dependent shortest path. The algorithm proposed here to minimize the travel time and provide a optimal path from source to destination with the facilities come in our path. We present here a algorithm on route planning which minimize the distance as well as time from source to destination.
\end{abstract}

\section{Keywords}

Location-based services, nearest query, Road network, and Route planning.

\section{INTRODUCTION}

In modern world everything seems to be faster and efficient for travelling. Route planning is an important for everyone so that everyone try to reach to destination as fast as possible .Route planning and its feature are important in many domains such as location based services, nearest neighbor and more so in GIS applications. For instance, when travelling from source to destination, although we can plan to cover some facility (eg petrol, grocery etc) prior to departure from source to destination with travel time

The user always prefers to travel in a less time and shortest path to reach to a destination. These two time dependent and shortest path are necessary for everyone via facility constraint.

Consider a figure 1 where a set of sample of a facility type (eg grocery, petrol etc), $\mathrm{F}=\{\mathrm{f} 1, \mathrm{f} 2, \mathrm{f} 3, \mathrm{f} 4, \mathrm{f5}\}$ is represent by stars on the road map. The minimum travel time and shortest path with one instance of each facility is shown by dark black line. The trip is influenced by many factors which is referred to as constraint on the network. Example of such constraint is user have known a time to reach a destination, other constraint have facilities which user cover within required time start from source to destination

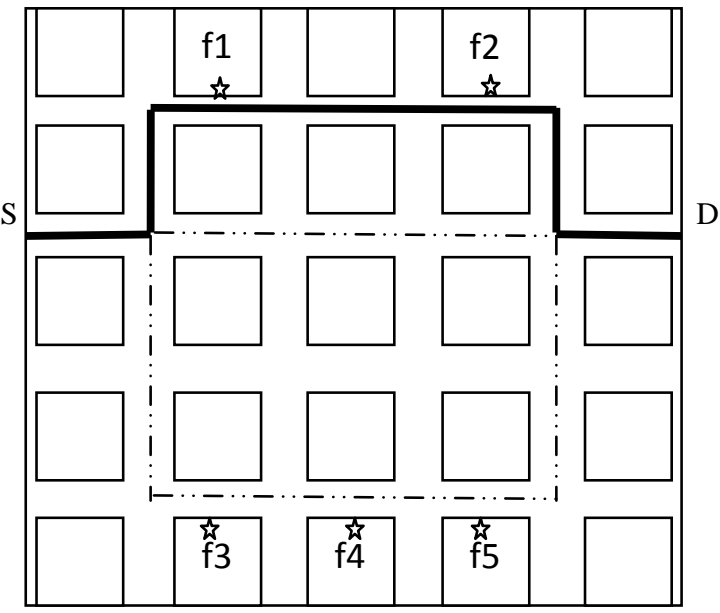

Fig. 1. An Example Network showing the Source to Destination routes

Above figure represent there are many path from source to destination via faculty. In one path there is three facility but it take more time to reach destination so it ignore, the other one is straight but there are not single facility so it also ignore, the last there are two facility and it take less travel time and shortest also so this path user can include in there traversing.

\section{Route Planning}

As everyone heard the term route planning not only heard but also applied in everyone's life. Before we go anywhere, we planned the route that we travel and list a facility which occur in our path. This can not only save our precious time but also expenditure.

Example:

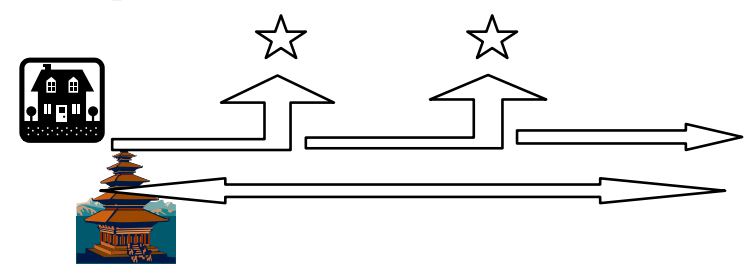

$20 \min$ 
Consider a real life example this cleared the views. User's home to college journey takes 20 minutes. Star represents the facility which comes in the way. College timing is $9.00 \mathrm{am}$. So user prefers to leave from home at 8.30 because the facility come in user way take $10 \mathrm{~min}$ extra. So user prefers to leave $30 \mathrm{~min}$ before to college. Therefore total $30 \mathrm{~min}$ is required to reach to college on time. This whole scenario represents a route planning.

This is important scenario in everyone's life as human tendency try to save time.

\section{RELATED WORK}

Many algorithm and methods have been proposed to find the nearest neighbor and shortest path. Nearest neighbor queries applied to find the closest object. Time dependent shortest path algorithm is there which is time oriented and give the optimal path. Time dependent shortest path find the optimal path from a source to destination. Route planning paper gives a concept to plan a path so that user can reach a destination as from planned way. Route planning with facility provide a user oriented application. In Route Nearest from a given path is minimum. IRNN applied constraint network problem as follows the shortest path from source to destination is picked up and then calculate whose facility deviation from this path is minimum. This procedure is repeated until all facility is covered.

Here basically focused on continuous monitoring of nearest neighbors (NN) in highly dynamic scenarios, where the queries and the data objects move frequently and arbitrarily. All existing methods, however, assume the Euclidean distance metric in their work. In this paper we study $k$-NN monitoring in road networks, where the distance between a query and a data object is determined by the length of the shortest and minimum path connecting them [1].

\section{DEFINITION}

This paper presents some solution for finding the optimal path for a road network .Optimal route means it take minimum travel time with shortest path. Always shortest path not necessary that it take less time. So we introduced a time dependent shortest path to go from source and reach a destination via facility.

\section{Assumptions:}

1. Start from a source node denoted as $\mathrm{S}$

2. Waiting time is ignored.

3. There should be at least one facility within the path.

\subsection{Terminology}

The terms used in this paper as :-

$\mathrm{V}$ denotes to nodes

D denotes destination

S denotes Source

$\mathrm{T}_{\mathrm{c}}$ denotes time constraint

$\mathrm{F}$ for facilities

\subsection{Time dependent Shortest Path Finding Algorithms}

There are many algorithms available for road distance between two points using a shortest path finding algorithm that are available [8, 11]. We use Dijkstra's single-source time dependent shortest path finding algorithm for this purpose [2].

There are basically two steps for shortest path as
Time refinement: In this we compute the earliest arrival time for every node. Dijkstra based time refinement is well refined till we reach destination

Path selection: The optimal path is computed using the path selection. To computed the optimal path from $v_{s}$ to $v_{e}$ we determine the predecessor of every node in backward from $\mathrm{v}_{\mathrm{e}}$ to $\mathrm{v}_{\mathrm{s}}$

\subsection{Problem Definition}

The problem is defined as follows:

Given:

1. A road network, $\mathrm{N}=\mathrm{N}(\mathrm{V}, \mathrm{E})$ where $\mathrm{V}$ is the number of nodes or vertices and $\mathrm{E}$ is the number of edges

2. A Source $\mathrm{S}$ and a Destination D

3. A set of instances of a facility type, $F=\left\{\mathrm{f}_{1}, \mathrm{f}_{2}, \ldots \mathrm{f}_{\mathrm{n}}\right\}$

4. Time Constraint $\mathrm{T}_{\mathrm{c}}$

Find:

A facility instance which occur in the path between source to destination.

Such that $\operatorname{dist}\left(\mathrm{S}, \mathrm{f}_{\mathrm{i}}\right)<=\mathrm{dc}$ and $\left[\operatorname{dist}\left(\mathrm{S}, \mathrm{f}_{\mathrm{i}}\right)+\operatorname{dist}\left(\mathrm{f}_{\mathrm{i}}, \mathrm{D}\right)\right]$ is minimum

Time Dependent Graph

A time dependent graph can be represented as $\mathrm{G}_{\mathrm{T}}(\mathrm{V}, \mathrm{E}, \mathrm{T})$

V set of vertices or source node

E set destination node

$\mathrm{T}$ is time interval

In this paper we concentrate on Travel Time that is departure time minus arrival time.

\section{Travel time=arrival time-departure time}

To understand this we explain with time dependent graph as

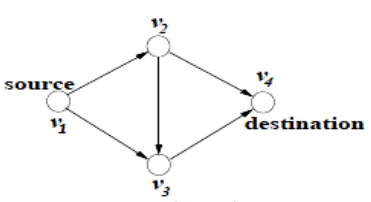

(a) $(V, E)$

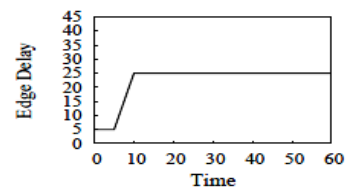

(c) $w_{1,3}(t)$

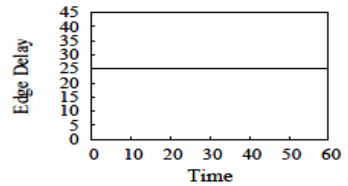

(e) $w_{2,4}(t)$

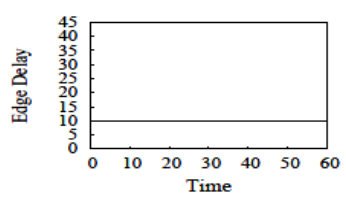

(b) $w_{1,2}(t)$

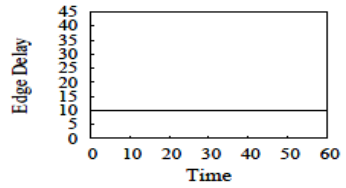

(d) $w_{2,3}(t)$

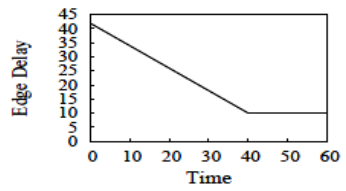

(f) $w_{3,4}(t)$
Example: A road network can be representing as time dependent graph. Above fig shows it has four nodes and five edges. There is also edge delay graph for each edge can be depicted in fig b,c,d,e,f graph The query as $\mathrm{G}_{\mathrm{T}}\left(\mathrm{v}_{1}, \mathrm{v}_{4},[0,60]\right)$. 
The optimal path is $\left(v_{1} v_{2} v_{3} v_{4}\right)$ as there edge delay is minimum

Starting time at $\mathrm{v} 1=20 \mathrm{~min}$

Arriving time at $\mathrm{v} 4=50 \mathrm{~min}$

Travel time $=30 \mathrm{~min}$

\section{APPROACHES}

There are some methods are developed which give optimal path from source to destination.

\section{Generalized approach with time algorithm}

This approach is applied to calculate the time dependent shortest path to all the facilities and then check the constraint.

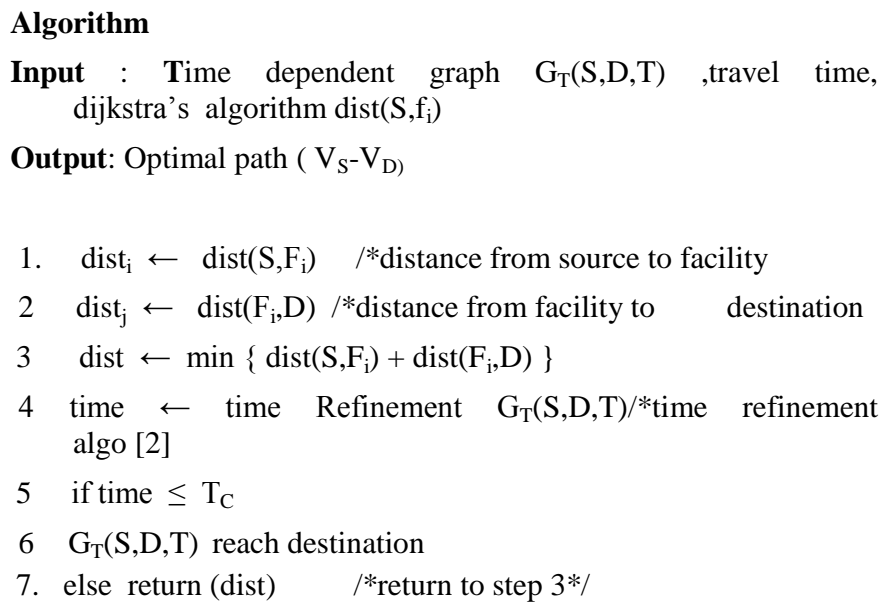

Here distance is calculated by dijsktra's algorithm from source to destination via facility. Now the distance calculated are pick up and put in ascending order. The distance which is on top is minimum distance.

Now we apply time refinement algorithm [2] which give us a time from source to destination via facility. As from above example time refinement calculate the time within vertices.

Now we check if time is less than time constraint (i.e the time in which user reach to destination) is satisfied than go to step 2 and select the next minimum distance and apply the same procedure else go to step 7 .

\section{Experimental Evaluation}

In this case, we present experimental evaluations to compare the above approach. We use synthetic networks dataset. Experiments are conducted to on different parameter and their impact can be seen by the graph. The parameters are number of queries, nodes, constraint distance, and constraint time. By conducting experiments we answered some questions.

1. Shortest distances do not mean your travel time is also less.

Answer is if edge delay is more than travel time is also more as seen in eg 2.1.

2. What is the effect of facility density and pre route planning's? All the experiments are carried on a 550-MHz Pentium PC machine with 256 megabytes main memory, running on Microsoft Windows.

\subsection{Datasets}

We report experimental results on synthetic. The synthetic data sets we used for our experiments, was generated using random function. The synthetic dataset used 50 nodes. In this we use number of facility are10. There is direct path from each vertex to every other vertex.

\subsection{Experimental Results}

Consider dataset D1. It has 100 nodes and 10 facilities. The graph is plotted as constraint distance with time and it denote as constraint distance increase facility density also increase.

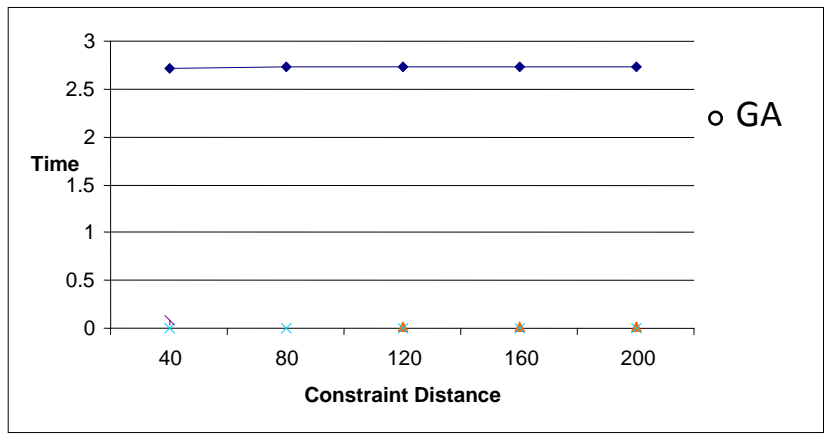

Fig. 3 Graph show the time versus constraint distance with generalized approaches

\section{CONCLUSION}

It is concluded from the above discussion that the route planning can be fast and efficient with the above algorithm. It reduces busy day to day schedule while covering most of the facility covering in the route. It can be implement in everyone's life i.e it is real life application. This algorithm can be work in opposite direction i.e if route planning has to be considered from destination to start point by covering at least one facility.

\section{ACKNOWLEDGEMENTS}

I wish to thank Mr. Atul Chaudhary, Asst. Professor, Computer Department whose guidance, teaching and certain suggestion provide me the timely valuable input which enhanced my knowledge.

\section{REFERENCES}

[1] K. Mouratidis, M.L. Yiu, D. Papadias, and N. Mamoulis, "Continuous Nearest Neighbor Monitoring in Road Networks," Proc. 32nd Int'l Conf. Very Large Data Bases (VLDB), pp. 43-54, 2006.

[2] Bolin.D and Xu.Y.J and Lu.Q, Finding time-dependent shortest path over large graphs. "International conference on Extending database technology", France, March 2008, pp. 205-216.

[3] F. Li, D. Chen, M. Hadjielefttherious, G. Kollios, S. Teng, On trip planning queries in spatial databases, SSTD, 2005.

[4] Xiaobin MA, Shashi Shekhar, Hui Xiong, Pusheng Zhang, Exploiting a Page-Level Upper Bound for 
Multi-Type Nearest Neighbor Queries, ACM-GIS, 2006.

[5] Athanasios Papoulis, S.Unnikrishna Pillai, Probability,Random Variables and Stochastic Process, 4th edition, McGrawHill, 2002.

[6] J. H. Rillings and R. J. Betsold. Advanced Driver Information Systems. IEEE Trans. on Vehicular Technology, 1991.

[7] M. Sharifzadeh, M. Kolahdouzan, C. Shahabi, The optomal sequenced route query, TR 05-840, CS, USC, January 2005.
[8] Jin Fu Leng Wen Zeng, “An Improved Shortest Path Algorithm for Computing One-to-One Shortest Paths on Road Networks", Fac. of Inf. Eng., China Univ. of Geosci., Wuhan, China, IEEE, 26 April 2010

[9] S. Shekhar, R. R. Vatsava, X. Ma, J. Yoo, Navigation Systems: A Spatial Database Perspective, In chapter 3 of the book, Location-Based Services, 2003.

[10] J. S. Yoo et al, S. Shekhar, In-Route Nearest Neighbor Queries, Geoinformatica, 9(2), June, 2005. 